\title{
Cultivo y composición bioquímica de diatomeas marinas (Bacillariophyta) de la Bahía de Santa Lucía, Acapulco, México
}

\section{Cultivation and biochemical composition of marine diatoms (Bacillariophyta) of Santa Lucía Bay, Acapulco, Mexico}

\section{Víctor A. Cervantes-Urieta르. Donaciano Pérez-Castro ${ }^{1}$, María A. Galeana-Parra ${ }^{1}$, Eustacio Ramírez-Fuentes ${ }^{2} \&$ Ma. Nieves Trujillo-Tapia ${ }^{3 *}$}

\author{
${ }^{1}$ Laboratorio de Biotecnología e Impacto Ambiental, Escuela Superior de Ciencias Ambientales, Universidad Autónoma de Guerrero, Carretera Cayaco-Puerto Marques Ejido \\ Llano Largo 56, 57 y 58, Acapulco, Guerrero, México. \\ ${ }^{2}$ Instituto de Recursos, Laboratorio de Biotecnología Ambiental, Universidad del Mar Puerto Ángel, Oaxaca, México. \\ ${ }^{3}$ Instituto de Ecología, Laboratorio de Biotecnología Ambiental, Universidad del Mar Puerto Ángel, Oaxaca, México. \\ *E-mail: nieves@angel.umar.mx
}

\section{RESUMEN}

El objetivo del presente estudio fue identificar, aislar, cultivar en sistemas de escalamiento discontinuos y evaluar el contenido bioquímico proximal de tres especies de diatomeas marinas nativas: Eupyxidicula turris, Trieres mobiliensis y Biddulphia alternans, de la Bahía Santa Lucia de Acapulco, México. La identificación de las especies se realizó con microscopia óptica binocular y microscopia electrónica de barrido. Así mismo, se evaluó el desarrollo de las tres especies a través de cinéticas de crecimiento y cuantificación del contenido proximal bioquímico (lípidos, proteínas, carbohidratos y cenizas). Las especies presentaron un crecimiento máximo de 2,1, 3,0 , y 8,5 células $\mathrm{mL}^{-1}$ y una velocidad especifica de crecimiento $>0,50$ días al final de la fase exponencial de la tercera escala; con un rendimiento de biomasa seca de 139,$6 ; 163,3$ y $219,9 \mathrm{mg}$ para E. turris, T. mobiliensis y $B$. alternans, respectivamente. El contenido de lípidos (11,1 $\pm 0,31$ a $11,5 \pm 0,18 \%)$, proteínas $(8,2 \pm 0,25$ a 8,8 \pm $0,35 \%)$, y carbohidratos $(10 \pm 0,22$ a $30 \pm 0,21 \%)$, fueron similares para las tres especies. Sin embargo, Biddulphia alternans presentó el mejor crecimiento celular ( $16 \times 10^{3}$ células diarias) y un mayor rendimiento de biomasa (219,9 $\left.\mathrm{mg} \mathrm{L}^{-1}\right)$; considerándola una especie de diatomea con potencial para posteriores investigaciones con fines de productividad primaria.

Palabras clave: metabolitos primarios, microalgas, producción.

\section{ABSTRACT}

The objective of this study was to identify, isolate and cultivate three species of marine diatoms in discontinuous scaling systems: Eupyxidicula turris, Trieres mobiliensis and Biddulphia alternans, from the Santa Lucia Bay of Acapulco, México. Identification of species was performed with binocular optical microscopy and scanning electron microscopy. Likewise, the development of three species through growth kinetics and quantification of proximal biochemical content (lipids, proteins, carbohydrates and ashes) was evaluated. The species showed a maximum growth of $2.1,3.0$, and 8.5 cells $\mathrm{mL}^{-1}$, and a specific growth rate $>0,50$ days, at the end of the exponential phase of the third scale; and a dry biomass yield of $139.6 ; 163.3$ and $219.9 \mathrm{mg}$ to E. turris, T. mobiliensis and B. alternans, respectively. Lipids content (11.1 \pm 0.31 to $11.5 \pm 0.18 \%)$, proteins $(8.2 \pm 0.25$ to $8.8 \pm 0.35 \%)$ and carbohydrates ( $10 \pm 0.22$ to $30 \pm 0.21 \%$ ), were similar for the three species. Nevertheless, Biddulphia alternans recorded better cell growth $\left(16 \times 10^{3}\right.$ daily cells) and higher biomass yield $\left(219.9 \mathrm{mg} \mathrm{L}^{-1}\right)$; considering it a diatom species with potential for further researches with biotechnological purposes.

Key words: microalgae, primary metabolites, production. 


\section{INTRODUCCIÓN}

Las diatomeas marinas son productores primarios fotoautótrofos y como primer eslabón en la cadena trófica cumplen una función esencial en los ecosistemas acuáticos; su amplia distribución y adaptabilidad en diversos ambientes marinos ha sido posible gracias a su gran diversidad metabólica como respuesta a diferentes escenarios ambientales (Infante et al. 2012). Las diatomeas han sido utilizadas en diversas investigaciones, particularmente como alimento vivo en acuicultura, sobre todo en cultivos de moluscos o etapas tempranas de peces (Vásquez et al. 2013). Algunas diatomeas marinas como Chaetoceros muerelli (McGinnis et al. 1997, Lemus et al. 2006, Panta-Vélez et al. 2016) y Skeletonema costatum (Pérez 1995, Zafra-Trelles et al. 2017, Zaouri et al. 2017), han sido utilizadas ampliamente como agentes nutricionales por aportar aproximadamente el $45,2 \pm 2,97 \%$ de proteínas, lípidos $14,39 \pm 1,02 \%$ y carbohidratos $18,34 \pm 2,08 \%$ en peso seco (Vásquez et al. 2010); esto revela la importancia económica que tienen las diatomeas marinas en la alimentación acuícola. Sin embargo, la utilización de microalgas particularmente diatomeas se ha limitado a solo unas cuantas especies, minimizando la búsqueda de nuevas microalgas como nuevas fuentes de obtención de agentes nutricionales.

En el Pacífico y Golfo de México se han documentado alrededor de 5.000 especies de diatomeas marinas (Hernández-Becerril 2014). En particular, para la Bahía Santa Lucía de Acapulco, el número reportado es de 88 géneros y 274 especies (Meave-del Castillo et al. 2012). No obstante, el estudio de estas especies de microalgas (incluidas las diatomeas) se ha visto limitado solo a estudios ecológicos (Rojas-Herrera et al. 2012). Aunado a esto, las especies de microalgas marinas pertenecientes al grupo de diatomeas centrales (Bacillariophyta): Trieres mobiliensis (J.W. Bailey) Ashworth and Theriot comb. nov. (antes Biddulphia mobiliensis), Biddulphia alternans J.W. Bailey y Eupyxidicula turris (Greville) Blanco \& Wetzel, comb. nov. (antes Stephanopyxis turris), han sido reportadas como parte principal de la comunidad y composición fitoplanctónica de la Bahía de Santa Lucía con densidades de 34 a 2647,56 y de 20 a 1162 células $L^{-1}$, para B. alternans, $S$. turris y B. mobiliensis respectivamente (MeaveDel Castillo et al. 2012; Rojas-Herrera et al. 2012; MorenoDíaz et al. 2015). En este sentido, Nájera-Arce et al. (2018) evaluaron la actividad biológica de diatomeas marinas, entre ellas B. mobiliensis encontrando compuestos como alcaloides, terpenos y aceites esenciales con actividad antibacteriana, también ha sido propuesta en otros países para la producción de biodiesel por poseer ácidos grasos de cadena larga (36,3 $\mathrm{mg} \mathrm{g}^{-1}$ de C16:1 y 10,5 $\mathrm{mg} \mathrm{g}^{-1}$ de C20) (Islam et al. 2013). La especie $S$. turris ha sido empleada como especie modelo para el estudio de la silificación en los océanos en relación a sus características genotípicas y fenotípicas (Finkel et al. 2010), así como trabajos sobre la influencia del hierro en la creación de frústulas silificadas de diatomeas marinas (Kaden et al. 2017). Por otra parte, B. alternans ha sido utilizada para comparar su contenido lipídico respecto a otras especies del orden Biddulphiophycidae (Fields \& Kociolek 2015). Sin embargo, aún se desconoce de investigación relacionada con el aislamiento y manejo in vitro de estas especies, particularmente en la Bahía de Acapulco; así como de parámetros como el pH, luz, salinidad, temperatura, nutrientes y otros factores que puedan favorecer su crecimiento, la estabilidad y viabilidad de sus moléculas en cultivos a pequeña, mediana y gran escala (Hernández-Pérez \& Labbé 2014, Matsumoto et al. 2017).

La evaluación de especies de microalgas marinas en condiciones de laboratorio y la implementación de sistemas de cultivo, son de suma importancia en el estudio fisiológico de las especies, ya que permite identificar las condiciones óptimas de crecimiento y producción de especies de microalgas locales. Particularmente, T. mobiliensis, B. alternans y E. turris poseen una amplia distribución subtropical, tropical, nerítica y oceánica (Meave-del Castillo et al. 2012) debido a su amplia adaptabilidad metabólica (Fogg 2001), lo que hace posible el manejo bajo diversos regímenes de cultivo y su aprovechamiento en términos biotecnológicos y económicos como una fuente de recurso natural sustentable para la obtención de compuestos primarios esenciales, como productos derivados, o bien en la acuicultura local como alimento vivo.

Por lo anterior, el objetivo del presente trabajo fue identificar, aislar, cultivar, evaluar el crecimiento celular y cuantificar el contenido bioquímico proximal de tres especies de diatomeas marinas: Trieres mobiliensis, Biddulphia alternans y Eupyxidicula turris presentes en la Bahía de Santa Lucía de Acapulco, México; con el propósito de caracterizar a las tres especies seleccionadas en este estudio y encontrar el mejor rendimiento a partir de cultivos discontinuos bajo condiciones de laboratorio.

\section{MATERIALES Y MÉTODOS}

MUESTREO, IDENTIFICACIÓN Y AISLAMIENTO DE LAS CEPAS ALGALES Se realizaron 10 arrastres planctónicos de febrero a marzo 2016 en 2 estaciones de muestreo dentro de la Bahía de Santa Lucía de Acapulco, Guerrero, México: 1) Morro del Fraile ( $\left.16^{\circ} 51^{\prime} 13^{\prime \prime} \mathrm{N}-99^{\circ} 52^{\prime 2} 27^{\prime \prime} \mathrm{W}\right)$ y 2) Terminal Marítima $\left(16^{\circ} 50^{\prime} 51^{\prime \prime} \mathrm{N}-99^{\circ} 53^{\prime} 59^{\prime \prime} \mathrm{W}\right)$, los arrastres fueron a $30 \mathrm{~cm}$ de profundidad con red de plancton tipo cono truncado de $1 \mathrm{~m}$ de largo, $52 \mathrm{~cm}$ de diámetro de boca y apertura de poro de $54 \mu \mathrm{m}$. Se obtuvo un total de 150 muestras recolectadas 
de $100 \mathrm{~mL}$ cada una, el material obtenido fue depositado en frascos herméticos de polietileno de $200 \mathrm{~mL}$ y trasladados al laboratorio evitando la exposición directa al sol.

La identificación de las especies fitoplanctónicas fue realizada mediante literatura especializada (Cupp 1943, Moreno et al. 1996, Hernández-Becerril 2000, OjedaRodríguez 2003, Round et al. 2007, Meave-del Castillo et al. 2012). Se utilizó microscopia óptica binocular de campo claro (Leica ${ }^{\circledR} 1349521 X$ ) y microscopia electrónica de barrido (Jeol ${ }^{\circledR}$ T300) para observar y corroborar la identificación de las especies aisladas. El aislamiento de las especies se llevó a cabo mediante micromanipulación directa utilizando una pipeta tipo Pasteur de punta alargada y microscopia óptica (Andersen 2005). Las especies aisladas fueron transferidas en tubos de ensayo con agua marina enriquecida con medio $\mathrm{F} / 2$, colocadas en un fotoperiodo 12:12 luz:oscuridad, temperatura media constante de $25 \pm 1^{\circ} \mathrm{C}$, irradiación de $55 \mu \mathrm{mol}$ fotón $\mathrm{m}^{-2} \mathrm{~s}^{-1}$ durante 15 días hasta obtener cultivos puros y monoclonales (Guillard 1973).

\section{CRECIMIENTO Y PRODUCCIÓN DE BIOMASA}

El crecimiento, escalamiento y producción de biomasa de Trieres mobiliensis (CT), Biddulphia alternans (CB) y Eupyxidicula turris (CE), se realizó en cultivos discontinuos, a partir de cultivos concentrados en agua marina (salinidad de 32-35 y $\mathrm{pH}$ de 7,8 -8) filtrada, esterilizada y enriquecida con medio $\mathrm{F} / 2$ a una concentración de $20 \mathrm{~mL} \mathrm{~L}^{-1}$, a partir de una solución (50X) (Guillard 1973). El escalamiento consistió de un inóculo al $10 \%$ en medio $\mathrm{F} / 2$ en la primera escala $(30 \mathrm{~mL}$ ), segunda escala al $10 \%$ de inóculo del primer escalamiento en medio $\mathrm{F} / 2$ $(100 \mathrm{~mL}$ ) y la tercera escala al $10 \%$ fue llevado a un volumen final de $1000 \mathrm{~mL}$ de medio $\mathrm{F} / 2$. Las condiciones de cultivo para las tres escalas fueron: fotoperiodo 12:12 luz:oscuridad, temperatura media constante de $25 \pm 1^{\circ} \mathrm{C}$, irradiación de 55 $\mu \mathrm{mol}$ fotón $\mathrm{m}^{-2} \mathrm{~s}^{-1}$ utilizando lámparas fluorescente de luz fría (Philips ${ }^{\circledR} 32$ Watt) y agitación manual cada 24 h durante 15 días (Guillard 1973).

La caracterización cuantitativa de las especies se llevó a cabo mediante concentración celular (DC), esta se determinó cada $24 \mathrm{~h}$ por triplicado mediante conteo directo para cada escala en cámara de Neubauer de $0,1 \mathrm{~mm}^{2}$, utilizando una muestra de $0,5 \mathrm{~mL}$ de cultivo previamente homogenizado y fijados con formol al 4\%, la concentración se calculó con la ecuación propuesta por Arredondo-Vega \& Voltolina (2007):

$D C=N \times 10^{4} \times$ Dil.

Dónde:

DC= Concentración celular (células $\mathrm{mL}^{-1}$ )
$\mathrm{N}=$ Promedio de células presentes en $1 \mathrm{~mm}^{2}(0,1 \mu \mathrm{L})$

$10^{4}=$ Factor de conversión de $0,1 \mu \mathrm{L}$ a $1 \mathrm{~mL}$

Dil= Factor de dilución

Así mismo, se determinó el número de divisiones por día con el cálculo de la velocidad específica de crecimiento $(\mu)$, tiempo de duplicación por día (TD) y producción diaria (PD) de cada especie evaluada durante el tiempo de cultivo y escalamiento (Andersen 2005).

La biomasa en peso seco (BPS) se obtuvo de la tercera escala a los 15 días de cultivo en la fase exponencial mediante auto-floculación por $24 \mathrm{~h}$ bajo las mismas condiciones de cultivo. Posteriormente la biomasa concentrada se separó por centrifugación a $6000 \mathrm{rpm}$ (en tubos Falcón de $50 \mathrm{~mL}$ ) y finalmente se liofilizó durante $11 \mathrm{~h}$ continuas a $-42^{\circ} \mathrm{C}$ y presión de $133 \times 10^{-3}$ bar, para realizar la caracterización bioquímica proximal correspondiente.

\section{EXTRACCIÓN Y CUANTIFICACIÓN DE LÍPIDOS TOTALES}

Para la extracción de lípidos totales se utilizó el método Bligh \& Dwyer (1959) modificado por Aguilar et al. (2011), utilizando de 5-10 mg de biomasa liofilizada. La cuantificación de lípidos se determinó gravimétricamente de acuerdo a la ecuación establecida por Arredondo-Vega \& Voltolina (2007):

\%Lípidos= $[(\mathrm{PTL}-\mathrm{PTV}) /(\mathrm{P})] \times 100$

Dónde:

$\mathrm{PTL}=$ Peso del tubo con lípidos (mg)

PTV= Peso del tubo vacío $(\mathrm{mg})$

$\mathrm{P}=$ Peso de la muestra $(\mathrm{mg})$

\section{DETERMINACIÓN DE PROTEÍNAS}

La cantidad de proteína fue calculada por micro Kjeldahl (Porta-Vales 2009). El valor de proteína total se calculó midiendo el contenido en nitrógeno $(\mathrm{N})$ en una muestra de $50 \mathrm{mg}$ de biomasa liofilizada, mediante digestión ácida 400 ${ }^{\circ} \mathrm{C}$ durante $2 \mathrm{~h}$. Posteriormente se realizó una destilación rápida utilizando $10 \mathrm{~mL}$ de ácido bórico saturado e indicador Thashiro. Finalmente se llevó a cabo una valoración con ácido clorhídrico $(\mathrm{HCl} 0,1 \mathrm{~N})$ para calcular el porcentaje de proteína con la siguiente ecuación:

\%Proteína $=[(\mathrm{mL} \mathrm{HCl}$ muestra- $\mathrm{mL} \mathrm{HCl}$ blanco $) \times \mathrm{N} \times \mathrm{MW} \times \mathrm{F}$ $x$ 100]/ Peso muestra (mg)

Dónde:

$\mathrm{N}=$ Normalidad del $\mathrm{HCl}$ utilizado en la valoración $(0,1 \mathrm{~N})$

$\mathrm{MW}=$ Peso molecular del $\mathrm{N}=14,007$

$\mathrm{F}=$ Factor de conversión empírico $=6,25$ 


\section{DETERMINACIÓN DE CENIZAS Y CARBOHIDRATOS}

El contenido en cenizas se obtuvo por calcinación utilizando de 30-50 mg de biomasa liofilizada sometidas a horno mufla a 600 ${ }^{\circ} \mathrm{C}$ durante $2 \mathrm{~h}$ para determinar por diferencia de peso (AOAC 1995). Por último, la cantidad de carbohidratos se obtuvo utilizando la ecuación establecida por Porta-Vales (2009):

\%Cenizas $=\mathrm{mg}$ cenizas $/ \mathrm{mg}$ muestra $* 100$

\%Carbohidratos= $100-\left(\%\right.$ Lípidos + \%Proteínas + \%Cenizas) ${ }^{(5)}$

\section{ANÁLISIS ESTADÍSTICO}

Para establecer diferencias entre los escalamientos, crecimiento y parámetros poblacionales de las especies, se realizó un análisis de varianza (ANOVA) de medias repetidas y posteriormente una prueba de Duncan para identificar diferencias entre las medias obtenidas. El rendimiento de BPS de las diferentes especies se determinó mediante un análisis ANOVA de una vía. Previo el análisis de varianza se realizó el test de normalidad de Shapiro-Wilk y el test de homogeneidad de varianza de Levene. Todas las pruebas se realizaron con un nivel de significancia del $95 \%(P=0,05)$ utilizando el software StatSoft ${ }^{\circledR}$ (STATISTICS 7).

\section{RESULTADOS}

\section{AisLAMIENTO E IDENTIFICACIÓN DE LAS CEPAS ALGALES}

Se lograron aislar e identificar tres especies de diatomeas marinas de la Bahía de Santa Lucía en Acapulco, Guerrero, México bajo las siguientes condiciones ambientales: salinidad de 32 a 35 unidades, $\mathrm{pH}$ de 8,0-8,3y una temperatura promedio de 30 a $31{ }^{\circ} \mathrm{C}$. Basados en las características morfológicas (ornamentaciones, procesos, vista cingular y valvar) que posee cada microalga, las especies de este estudio pertenecen a la división Bacillariophyta (diatomeas céntricas): Trieres mobiliensis se encontró en la primera estación de muestreo (Morro del Fraile); mientras que Biddulphia alternans y Eupyxidicula turris se encontraron en la segunda estación (Terminal Marítima). Por otra parte, la micromanipulación y cultivo in vitro de las tres microalgas permitieron una adaptación y viabilidad de las cepas para cultivo a los 8 días después del aislamiento en agua marina enriquecida con medio $F / 2$ en un volumen inicial de $30 \mathrm{~mL}$ (Fig. 1, fila S1, columnas CB, CT y CE). Las células de $T$. mobiliensis presentan un tamaño de $235 \times 175 \mu \mathrm{m}$ con alargamiento en sedas laterales (Fig. 1, fila SI, columna CT-2 y CT-3), así como la disminución o ausencia de sedas centrales, terminaciones valvares y plastos. En la segunda semana después del aislamiento, T. mobiliensis presentó formaciones de cadenas celulares de máximo dos células y un aumento en su tamaño de $358 \times 105 \mu \mathrm{m}$ (Fig. 1 fila SII, columna CT).

Las microfotografías mediante microscopía electrónica de barrido (MEB) mostraron una banda ecuatorial en vista valvar en forma oval a oblonga con una ligera elevación respecto a los polos de la célula, con sedas o espinas centrales y laterales característica de esta especie (Fig. 2, fila CT, columna I y II). La especie B. alternans, tiene un tamaño promedio de 39,42 x 42,29 mm y cadenas más largas ( 2 a 4 células) que T. mobiliensis. Así mismo, posee una vista valvar es de forma triangular, vista singular de forma cuadrada y vista dorsal trapezoidal (Fig. 1, fila SI y SII, columnas CB-2 a CB-4). Posee terminaciones valvares con un poro circular y cíngulo plano de vista dorsal (Fig. 2, fila CB, columna I y II) indicando que la especie es morfológicamente estable durante los 15 días de cultivos. En lo que respecta a E. turris, se observó un marcado alargamiento celular de forma cilíndrica (560 × 40 $\mu \mathrm{m}$ ) (Fig. 1, fila S0 y SI, columna CE-1 y CE-2). La formación de cadenas (5 a 15 células por cadena) es mucho más larga con respecto a las otras dos especies (Fig. 1, fila SII, columna CE-3). Las observaciones MEB mostraron un eje valvar de forma campanulada a coronada con alargamiento de espinas centrales de 5,97 a 12,34 mm (Fig. 2, fila CE, columna I y II).

\section{DENSIDAD POBLACIONAL Y PRODUCCIÓN DE BIOMASA}

El cultivo de cada una de las especies aisladas se inició con una densidad de 0,6 a 4,0 × $10^{4}$ células $\mathrm{mL}^{-1}$ en el primer escalamiento. Después de cinco días de cultivo se obtuvieron valores máximos de 1,6 a 4,3 × $10^{4}$ células $\mathrm{mL}^{-1}$, presentando diferencias estadísticamente significativas entre los tres escalamientos $\left(F_{(20,225)}=1,6433 ; P<0,05\right)$ (Fig. 3). La velocidad específica de crecimiento $(\mu)$ durante el primer escalamiento osciló entre 0,25 $\mu$ (CE-1), 0,33 $\mu$ (CB-3) y 0,43 $\mu$ (CT-2) (Tabla 1). Durante el segundo escalamiento, la cepa que presentó una mejor adaptación fue la CT-2, alcanzando una $\mu$ de 0,26 por día; mientras que la cepa CB-3, alcanzó una $\mu$ de 0,05 , hasta las $72 \mathrm{~h}$, lo que limitó su crecimiento. Sin embargo, la cepa CE-1, no se adaptó en este escalamiento, declinando su crecimiento totalmente $(-0,03 \mu)$. En la tercera escala, el cultivo de las tres cepas se inició con una concentración celular de $0,5 \times 10^{4}$ células $\mathrm{mL}^{-1}$, las cepas mostraron una mejor adaptación y crecimiento a las condiciones de cultivo: nutrientes, fotoperiodo y temperatura. E. turris (CE1), presentó un incremento del $20 \%$ en su crecimiento con respecto al primero y segundo escalamiento y recuperó su $\mu(0,29)$ en este tercer escalamiento. T. mobiliensis (CT2) mostró una mejor adaptación en los tres escalamientos, mantuvo su $\mu$ entre 0,43 a 0,34. B. alternans (CB-3) por su parte presentó los valores máximos de concentración celular de $8,56 \times 10^{4}$ células $\mathrm{mL}^{-1}$ y un crecimiento de 0,56 ; tres veces más comparado con T. mobiliensis $\left(2,86 \times 10^{4}\right.$ células $\left.\mathrm{mL}^{-1}\right)$. 


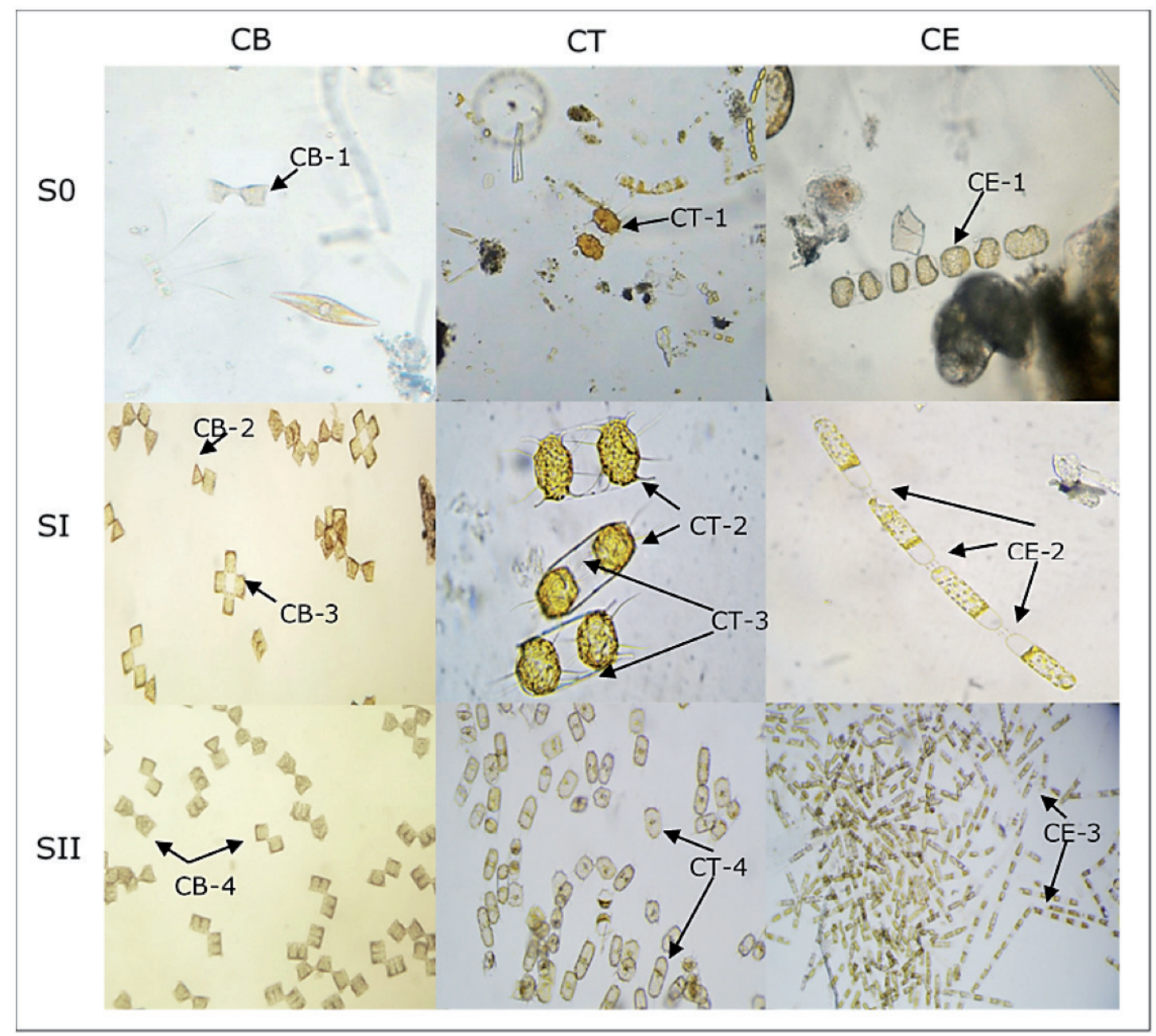

FIgura 1. Semanas del cultivo: (SO) semana cero; (SI) semana uno; (SII) semana dos. Biddulphia alternans (CB): B. alternans en medio natural (CB-1); Vista triangular y cuadrangular/séptimo día de cultivo observada a 40x (CB-2 y CB-3). Trieres mobiliensis (CT): Plastos presentes en toda la célula (CT-1); Espinas laterales en forma curvada y centrales superiores prolongadas (CT-2); Unión de espinas centrales y laterales entre células (CT-3). Eupyxidicula turris (CE): Células circulares a semicirculares con estrecha separación entre células (CE-1); Espinas polares (CE-2). Cadenas de B. alternans (CB-4), T. mobiliensis (CT-4) y E. turris (CE-3) a la segunda semana de cultivo, vistas a 10x. / Cultivation weeks: (SO) zero week; (SI) week one; (SII) week two. Biddulphia alternans (CB): B. alternans in natural medium (CB-1); Triangular and quadrangular view / seventh day of culture observed at 40x (CB-2 and CB-3). Trieres mobiliensis (CT): Plastids present throughout the cell (CT-1); Lateral spines in curved form and prolonged upper centrals (CT-2); Union of central spines and controls between cells (CT-3). Eupyxidicula turris (CE): circular to semicircular cells with close separation between cells (CE-1); Polar spines (CE-2). Chains of B. alternans (CB-4), T. mobiliensis (CB-4) and E. turris (CE-3) at the second week of cultivation, seen at 10x.

TABLA 1. Parámetros poblacionales de diatomeas marinas cultivadas en diferentes escalas. / Population parameters of marine diatoms cultivated in different scales.

\begin{tabular}{|c|c|c|c|c|c|c|c|c|c|}
\hline \multirow[b]{2}{*}{ Especies } & \multicolumn{3}{|c|}{ Escala 1} & \multicolumn{3}{|c|}{ Escala 2} & \multicolumn{3}{|c|}{ Escala 3} \\
\hline & $\begin{array}{l}\mu \\
\text { (d) }\end{array}$ & $\begin{array}{l}\text { TD } \\
\text { (d) }\end{array}$ & $\begin{array}{c}\text { PD } \\
\left(\text { Cél } \mathrm{mL}^{-1} \text { ) }\right.\end{array}$ & $\begin{array}{l}\mu \\
\text { (d) }\end{array}$ & $\begin{array}{l}\text { TD } \\
\text { (d) }\end{array}$ & $\begin{array}{c}\text { PD } \\
\left(\text { Cél } \mathrm{mL}^{-1}\right)\end{array}$ & $\begin{array}{c}\mu \\
\text { (d) }\end{array}$ & $\begin{array}{l}\text { TD } \\
\text { (d) }\end{array}$ & $\begin{array}{c}\text { PD } \\
\left(\text { Cél } \mathrm{mL}^{-1}\right)\end{array}$ \\
\hline Eupyxidicula turris & 0,25 & 1,19 & 17333,33 & $-0,03$ & 1,21 & $-1000,0$ & 0,29 & 1,16 & 3333,33 \\
\hline Trieres mobiliensis & 0,43 & 1,64 & 7666,67 & 0,26 & 1,45 & 3666,67 & 0,34 & 1,41 & 4666,67 \\
\hline Biddulphia alternans & 0,33 & 1,36 & 17333,33 & 0,05 & 0,77 & 2666,67 & 0,56 & 1,9 & 16000,0 \\
\hline
\end{tabular}

$\mu=$ Velocidad específica de crecimiento, $T D=$ Tiempo de duplicación, $P D=$ Producción diaria, (d)= Días 


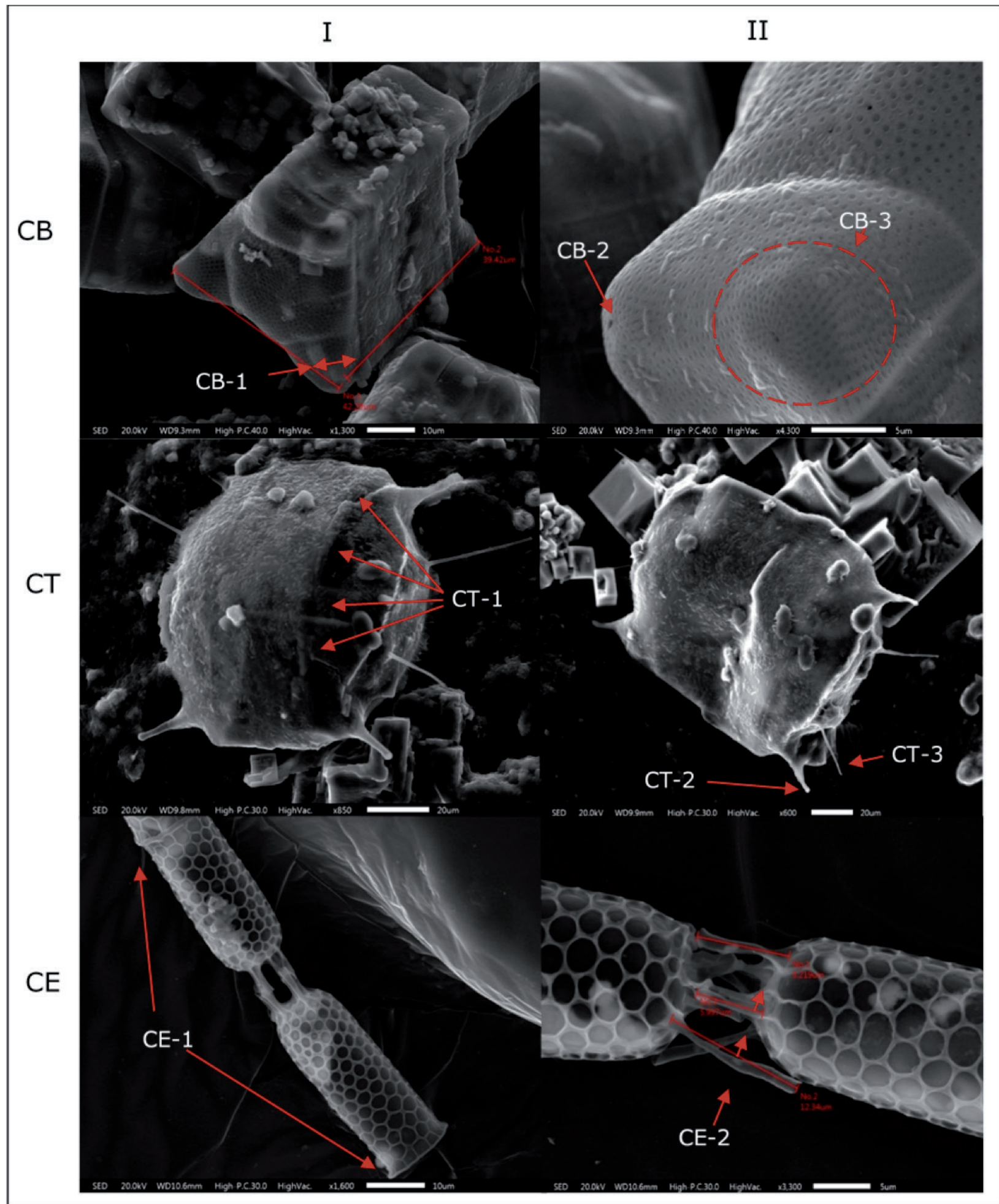

Figura 2. Microfotografías MEB de Biddulphia alternans (CB): Longitudes de 39-42 $\mu \mathrm{m}$ (CB-1); Terminaciones valvares (CB-2 y CB-3). Trieres mobiliensis (CT): Eje valvar con elevación (CT-2); Prolongaciones centrales y laterales (CT-2 y CT-3). Eupyxidicula turris (CS): Eje valvar en forma cilíndrica (CS-1- I), Espinas centrales (CS-2- II). / MEB micrographs of Biddulphia alternans (CBa): Length of 39-42 $\mu$ m (CBa-1); Valvar endings (CBa-2 and CBa-3). Trieres mobiliensis (CB): Valve axis with elevation (CB-2); Central and lateral prolongations (CB-2 and CB-3). Eupyxidicula turris (CE): Valve axis in cylindrical shape (CT-1), Central spines (CT-2-II). 

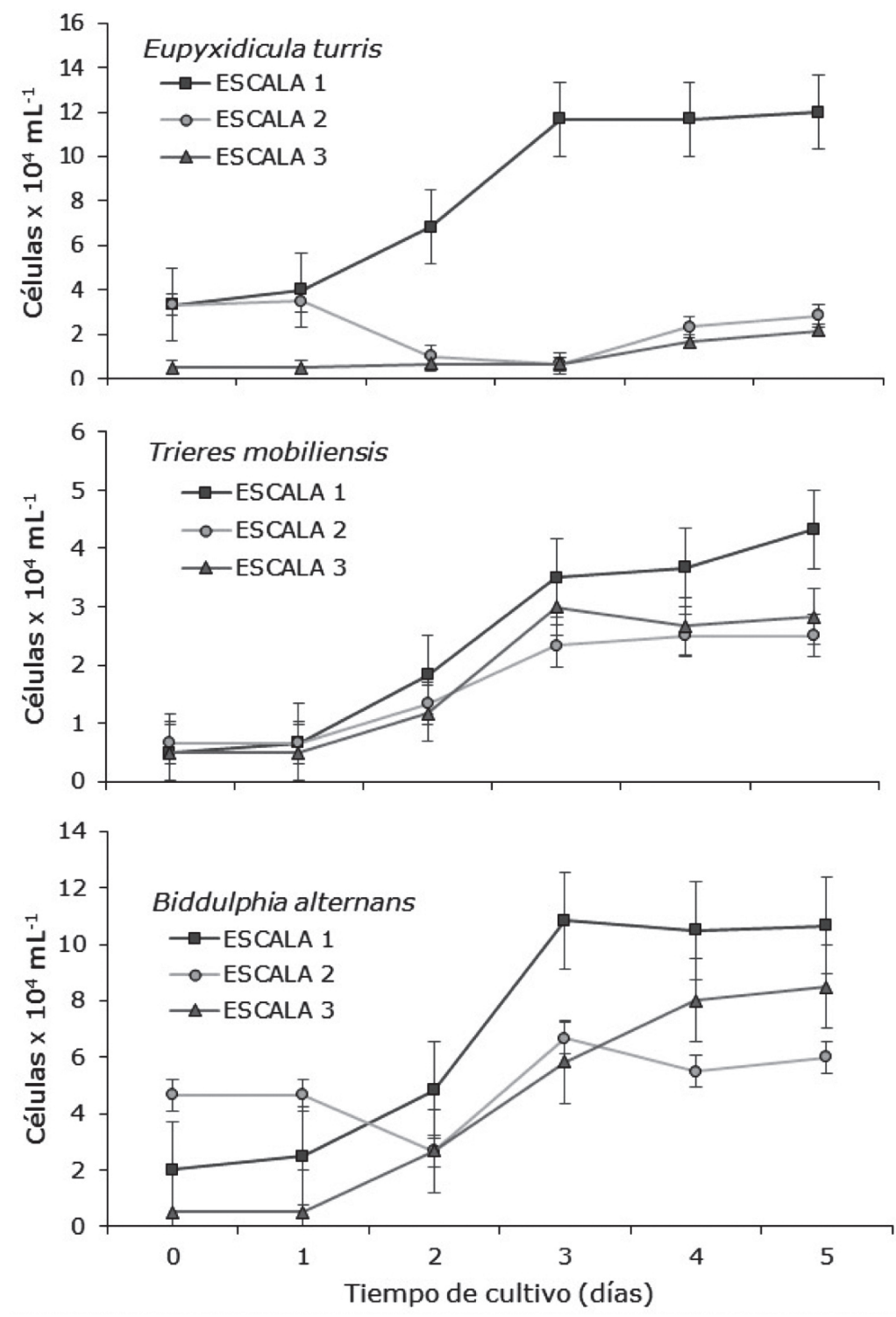

FiguRA 3. Escalamiento y cinética de crecimiento de tres diatomeas marinas de la Bahía de Santa Lucía. Los datos representan el promedio de los recuentos celulares por triplicado \pm desviación estándar. / Scaling and growth kinetics of three marine diatoms of the Santa Lucia Bay. Data represent the average of cell counts in triplicate \pm standard deviation.

TABLA 2. Biomasa en peso seco (BPS) y concentración celular en cultivos semicontinuos. / Biomass in dry weight (BPS) and cell density in semicontinuous cultures.

\begin{tabular}{|c|c|c|c|c|c|c|c|}
\hline \multirow[b]{2}{*}{ Especies/Escalas } & \multicolumn{3}{|c|}{ 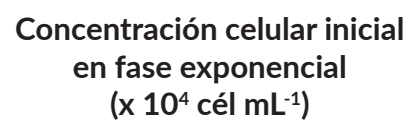 } & \multicolumn{3}{|c|}{$\begin{array}{l}\text { Concentración celular final en } \\
\text { fase exponencial } \\
\left(\times 10^{4} \text { cél } \mathrm{mL}^{-1}\right)\end{array}$} & \multirow{2}{*}{$\begin{array}{c}\text { Biomasa en } \\
\text { peso seco } \\
\left(\mathrm{mg} \mathrm{L}^{-1}\right)\end{array}$} \\
\hline & 1 & 2 & 3 & 1 & 2 & 3 & \\
\hline Eupyxidicula turris & 4,0 & 1,0 & 0,6 & 11,6 & 2,8 & 2,1 & 139,6 \\
\hline Trieres mobiliensis & 0,6 & 0,6 & 0,5 & 4,3 & 2,5 & 3,0 & 163,3 \\
\hline Biddulphia alternans & 2,5 & 2,6 & 0,5 & 10,8 & 6,6 & 8,5 & 219,9 \\
\hline
\end{tabular}


TABLA 3. Composición bioquímica porcentual de tres diatomeas cultivadas en condiciones de laboratorio. / Percent biochemical composition of three diatoms cultivated under laboratory conditions.

\begin{tabular}{|c|c|c|c|c|}
\hline Especies & $\begin{array}{c}\text { Lípidos Totales } \\
\%\end{array}$ & $\begin{array}{c}\text { Proteínas } \\
\%\end{array}$ & $\begin{array}{c}\text { Carbohidratos } \\
\%\end{array}$ & $\begin{array}{c}\text { Cenizas } \\
\%\end{array}$ \\
\hline Eupyxidicula turris & $11,2 \pm 0,45$ & $8,8 \pm 0,35$ & $30 \pm 0,21$ & $50 \pm 0,12$ \\
\hline Trieres mobiliensis & $11,1 \pm 0,31$ & $8,9 \pm 0,23$ & $10 \pm 0,22$ & $70 \pm 0,18$ \\
\hline Biddulphia alternans & $11,5 \pm 0,18$ & $8,2 \pm 0,25$ & $17 \pm 0,30$ & $63 \pm 0,13$ \\
\hline
\end{tabular}

Los valores mayores de concentración celular máxima y de $\mu$ fueron obtenidos en B. alternans (Tabla1), considerándose la especie de diatomea que mejor se adaptó al escalamiento. Esto se vio reflejado en la producción de biomasa con un incremento de 1,3 veces más que T. mobiliensis (CT-2) (Tabla 2). Los valores menores de concentración celular, de velocidad de crecimiento $(\mu)$, así como de producción de biomasa los presentó E. turris.

\section{COMPOSICIÓN BIOQUÍMICA}

La producción de lípidos y proteínas de las tres especies de diatomeas marinas no presentaron diferencias significativas (Tabla 3); el valor promedio de lípidos y proteínas fue de 11,2 y $8,6 \%$, respectivamente. Por lo que respecta al contenido de carbohidratos, el valor fue similar para T. mobiliensis (10 \pm $0,22 \%$ ) y $B$. alternans $(17 \pm 0,30 \%)$. E. turris (CE-1) produjo tres veces más carbohidratos comparada con las demás especies, a pesar de presentar un peso seco de $16 \%$ menos que $T$. mobiliensis (CT-2) y $57 \%$ menos que B. alternans (CB-3); las diferencias en peso se vieron reflejadas en el contenido de cenizas (Tabla 3).

\section{DISCUSIÓN}

Las diatomeas E. turris, T. mobiliensis y B. alternans fueron identificadas y recolectadas superficialmente durante los meses de febrero a marzo 2016, dato similar a los reportado por Nájera-Arce et al. (2018) quienes identificaron y aislaron en el periodo de diciembre del 2015 a marzo 2016 a Chaetoceros curvisetus, Asterionella japonica y Biddulphia mobiliensis para la Bahía de Santa Lucía de Acapulco. Mientras que Meave-del Castillo et al. (2012), identificaron a Stephanopyxis turris (ahora E. turris), Biddulphia alternans, B. biddulphiana y B. tridens de febrero a mayo del 2010. En ambos estudios se reportaron salinidad del agua de mar de $32 \pm 1$, y una temperatura de 31 ${ }^{\circ} \mathrm{C}$. Sin embargo, Moreno-Díaz et al. (2015) y Rojas-Herrera et al. (2012) en el periodo de febrero a mayo del 2010 al 2012 reportaron una mayor presencia de 54 dinoflagelados, ambos en época de surgencias. La presencia de las especies estudiadas en el presente trabajo es atribuida a las variaciones oceanográficas y climatológicas ocasionadas por el evento "El
Niño (EN) del Pacífico tropical del este, el cual se presenta en ciclos irregulares de 2 a 7 años (Hanley et al. 2003); favoreciendo la sucesión de las especies que conforman el fitoplancton, de acuerdo a los rangos óptimos de temperatura y las condiciones de su hábitat natural. En este estudio las diatomeas E. turris, B. alternans y T. mobiliensis son especies que prevalecen preferentemente en temperaturas cálidas $\left(\geq 30^{\circ} \mathrm{C}\right.$ ) sobre aguas costeras, características atribuibles al fitoplancton nerítico de la Bahía de Acapulco confirmando lo reportado por Ojeda-Rodríguez (2003), Ferrario et al. (2012) y Meave-del Castillo et al. (2012). Sin embargo, Richmond (2004), menciona que la temperatura en la cual un gran número de microalgas incluidas las diatomeas presentan una alta tasa reproductiva y una mejor adaptación, es diferente a la temperatura en las que los procesos bioquímicos transforman más eficientemente los nutrientes y su actividad fotosintética.

Las tres especies de diatomeas estudiadas presentaron un crecimiento diferente en los tres escalamientos; presentaron una respuesta favorable para la producción de biomasa con el medio $\mathrm{F} / 2$; sin embargo, la mejor producción celular fue de la más pequeña $B$. alternans (CB-3) en comparación con las de mayor tamaño T. mobiliensis y E. turris (CT-2 y CE-1), debido a que las diatomeas de menor tamaño tienen una mayor relación superficie/volumen, fenómeno que les permite absorber nutrientes a una mayor velocidad (Fogg 1975); derivando en una mayor cantidad de energía para la formación de nuevas células (Barreto \& Velasco 2014).

La temperatura juega un papel muy importante en el aislamiento y crecimiento celular de microalgas, la temperatura óptima reportada es de 20 a $25^{\circ} \mathrm{C}$ (Adenan et al. 2013, Barreto \& Velasco 2014, Moronta et al. 2006). El incremento de la tasa metabólica y fotosintética de las diatomeas y de algunas otras microalgas es proporcional a la temperatura (De Nicola 1996) y se refleja en la captación de nutrientes del medio para realizar adecuadamente los procesos metabólicos involucrados en el crecimiento celular (López-Elías 2002, Barreto \& Velasco 2014). B. alternans y T. mobiliensis presentaron un crecimiento consistente, con las características del medio de cultivo, irradiancia, fotoperiodo y temperatura; sin embargo, la poca capacidad de crecimiento de $E$. turris fue posiblemente a que la temperatura utilizada 
no fue la óptima para su crecimiento. Al respecto Renaud et al. (2002), reportan la velocidad de crecimiento $(\mu)$ de cinco microalgas tropicales en un intervalo de temperatura de 27 a $35{ }^{\circ} \mathrm{C}$; para el caso de Isochrysis sp., Rhodomonas sp. y Cryptomonas sp. presentaron un mejor crecimiento a intervalos de temperatura de 25 a $30^{\circ} \mathrm{C}$, mientras que algunas especies del genero Chaetoceros el intervalo de temperatura fue de 33 a $35^{\circ} \mathrm{C}(\mu=0,78)$, en dicho intervalo, Cryptomonas sp. presentó una $\mu=-0.01$, Isochrysis sp. y Rhodomonas sp. presentan valores de $\mu=-0,04$ y $-0,01$, respectivamente. En nuestro estudio $E$. turris a $25^{\circ} \mathrm{C}$ presentó una $\mu=-0,03$; lo que sugiere de temperaturas menores durante su cultivo, debido a que esta especie tiene una mayor afinidad a ecosistemas templados (Hallegraeff 2010), por lo que el efecto de la temperatura también se ve reflejado en el crecimiento celular y composición química intracelular (Thompson et al. 1992). Renaud et al. (2002) reportaron una producción de proteínas (31,5 a 64,1\%), lípidos (8,0 a $21,7 \%)$ y carbohidratos (4,4 a $14,2 \%$ ) con temperaturas de 25 a $35^{\circ} \mathrm{C}$ y una salinidad de 25 . Si bien, las diatomeas tienen un amplio rango de adaptación y tolerancia a la salinidad, Leal et al. (2013) reportan variación en el contenido de proteínas (28 a 19\%), lípidos (26 a 19\%) y carbohidratos (11 a 8\%) a diferentes concentraciones de salinidad (20 a 40) en dos especies de diatomeas bentónicas; también observaron que conforme aumentó la salinidad (35 a 40 ) el contenido de cenizas fue $>50 \%$. Las especies $E$. turris, B. alternans y T. mobiliensis muestran porcentajes altos de cenizas en peso en seco (50 a 70\%), nuestros resultados son similares a los encontrados por Porta-Vales (2009) en la composición de cenizas en diferentes fases de cosecha de las diatomeas Amphora sp., Navicula incerta, Nitzschia sp. y Proschkinia sp. Si bien Renaud et al. (2002) reportan que a temperaturas superiores de los $27^{\circ} \mathrm{C}$, algunas microalgas no fueron capaces de sobrevivir (e.g., Cryptomonas sp. a $33^{\circ} \mathrm{C}$ ), hubo especies que mantuvieron su producción de proteínas, lípidos y carbohidratos constante.

La irradiancia y la disponibilidad de nutrientes son factores que afectan de manera directa el crecimiento y composición celular de las diatomeas (Sarthou et al. 2005). Bender et al. (2012), evaluaron la interacción de tres fuentes de nitrógeno (nitrato, amonio y urea) con tres intensidades lumínicas (irradiancias de 50, 190 y $400 \mu \mathrm{mol}$ photon $\mathrm{m}^{-2} \mathrm{~s}^{-1}$ ), sobre la fisiología celular y expresión genética de Thalassiosira pseudonana y reportaron que la disponibilidad de nutrientes (relación C:N) y la intensidad de luz no fueron limitantes para el crecimiento celular; sin embargo al aplicar una irradiancia saturante $\left(190 \mu \mathrm{mol}\right.$ photon $\left.\mathrm{m}^{-2} \mathrm{~s}^{-1}\right)$, reportaron mejores tasas de crecimiento (d) de 0,99, 1,22 y 1,34 asociadas a las fuentes de nitrógeno (nitrato, amonio y urea respectivamente). En este sentido la expresión genética (abundancia de los transcritos asociados al metabolismo del nitrógeno), la interacción entre la fuente de nitrógeno, la intensidad de luz, el ciclo de luz y oscuridad si fue afectada significativamente $(P<0,05)$ durante el tiempo de cultivo y escalamiento, demostrando la plasticidad genética de las diatomeas para adaptarse a las variaciones ambientales del medio donde se desarrolla y mantener un crecimiento constante. Así mismo, la irradiancia asociada a diversas temperaturas y tipos de nutrientes también son factores determinantes para el cultivo de este tipo de microalgas (diatomeas).

Por otro lado, la sobrevivencia de las diatomeas y de otras microalgas fotosintéticas a diferentes condiciones ambientales, es el resultado de la activación de diferentes estrategias metabólicas para la producción de una diversidad de compuestos químicos (Barros et al. 2005). La alta producción de proteínas, lípidos, carbohidratos y pigmentos pude lograrse manipulando las condiciones ambientales y la genética de las microalgas logrando producir en rangos de 2 al $75 \%$ en peso seco. Esta variedad de compuestos tiene aplicación como alimento vivo, en la industria alimenticia, farmacológica (antiinflamatoria, antiobesidad y neuroprotectora) y en cosmetología. Hasta la fecha, no hay reportes sobre la evaluación bioquímica de las especies $E$. turris, B. alternans y T. mobiliensis; y por lo tanto, con el presente trabajo, se inició la investigación en un área de aprovechamiento no explorado en la Bahía de Acapulco. Sin embargo, el éxito en cultivos de diatomeas a pequeña, mediana o grande escala depende en general de su tamaño, resistencia, flotabilidad, capacidad de crecimiento, y de las condiciones a las que se someten (temperatura, fotoperiodo, calidad y cantidad de luz). Además, la fuente de carbono y nitrógeno, salinidad, $\mathrm{pH}$ y la fase de crecimiento para su cosecha, tienen influencia directa sobre la tasa de crecimiento y su composición bioquímica.

Durante el cultivo de microalgas en este estudio, las diatomeas Eupyxidicula turris (CE) y Trieres mobiliensis (CT), presentaron cambios en su morfología celular, pero el contenido bioquímico y crecimiento fueron similares durante su crecimiento y escalamiento. Biddulphia alternans (CB) presentó una tasa de crecimiento constante $(\mu>0,50)$, sin cambios morfológicos celulares y una rápida adaptabilidad para crecer in vitro a diferentes regímenes de cultivos, además de poseer un tamaño menor a los $42 \mu \mathrm{m}$ respectivamente. Por lo anterior, B. alternans es una especie prometedora para la producción como alimento vivo en sistemas acuícolas.

\section{AGRADECIMIENTOS}

Se agradece a la Universidad del Mar por el acceso a sus instalaciones para la realización experimental del presente 
estudio, al Dr. Oscar Talavera Mendoza, a la M.C. Jazmín López Díaz por el proceso fotográfico en el Laboratorio de Microscopía Electrónica de Barrido y Microanálisis de la Universidad Autónoma de Guerrero y a los evaluadores externos por las observaciones realizadas para la mejora del presente manuscrito.

\section{REFERENCIAS}

Adenan, N.S., Yusoff, F.M., Shariff, M. 2013. Effect of salinity and temperature on the growth of diatoms and green algae. Journal of Fisheries and Aquatic Science 8: 397-404. DOI: $10.3923 /$ jfas.2013.397.404

Aguilar, S.C., Chang, A.l., Tenorio G.L., Ynga, H.G., Oscanoa H.A., Flores R.L. 2011. Determinación de la biomasa microalgal potencialmente acumuladora de lípidos para la obtención de combustible. Instituto del Mar del Perú. Lima. 21 pp. URL: http://www.imarpe.pe/imarpe/archivos/informes/ proc_culti_microalgas.pdf

Andersen, R. 2005. Traditional Microalgae Isolation Techniques. In: Andersen, R. (Ed.) Algal Culturing Techniques: 83-100. Academic Press, Amsterdam.

AOAC. 1995. Official Methods of Analysis. Association Official Analytical Chemists International, Virginia 4: 1-30.

Arredondo-Vega, B.O., Voltolina, D. 2007. Manual de Métodos y Herramientas Analíticas en la Evaluación de la Biomasa Microalgal. Centro de Investigaciones Biológicas del Noroeste, Baja California Sur. 97 pp.

Barreto, H.A., Velasco, L.A. 2014. Aislamiento y cultivo de microalgas bentónicas del Caribe colombiano bajo diferentes condiciones de temperatura. Intropica 9: 2332.

Barros, M.P., Pinto, E., Sigaud-kutner, T.C.S., Cardozo, K.H.M., Colepicolo, P. 2005. Rhytmicity and oxidative/nitrosative stress in algae. Biological Rhythm Research 36: 67-82.

Bender, S.J., Parker, M.S., Armbrust, E.V. 2012. Coupled Effects of Light and Nitrogen Source on the Urea Cycle and Nitrogen Metabolism over a Diel Cycle in the Marine Diatom Thalassiosira pseudonana. Protist 163(2): 232251.

Bligh, E.G., Dwyer, W.J. 1959. A rapid method of total lipid extraction and purification. Canadian Journal of Biochemistry and Physiology 37: 911-917.

Cupp, E.E. 1943. Marine planckton diatoms of the west coast of North America. Institution of Oceanography of the University of California Press, San Diego. 138 pp.

De Nicola, D.M. 1996. Periphyton responses to temperature at different ecological levels. In: Stevenson, R.J., Bothwell, M.L., Lowe, R.L. (Eds.) Algal Ecology, Freshwater Benthic
Ecosystems: 150-166. Academic Press, San Diego.

Ferrario, M.E., Gastón, O.A., Cefarelli, A.O., Fabro, E., Vernet, M. 2012. Stephanopyxis species (Bacillariophyceae) from shelf and slope waters of the Argentinean Sea: Ultrastructure and distribution. Nova Hedwigia 96: 249263. Doi: 10.1127/0029-5035/2012/0077

Fields, F.J., Kociolek, J.P. 2015. An evolutionary perspective on selecting high-lipid-content diatoms (Bacillariophyta). Journal of Applied Phycology 27(6): 2209-2220. Doi: 10.1007/s10811-014-0505-1

Finkel, Z.V., Matheson, K.A., Regan, K.S., Irwin, A.J. 2010. Genotypic and phenotypic variation in diatom silicification under paleo-oceanographic conditions. Geobiology 8: 433-445.

Fogg, G.E. 1975. Algal cultures and phytoplankton ecology. The University of Wisconsin Press: 175. Wisconsin.

Fogg, G.E. 2001. Algal Adaptation to Stress - Some General Remarks. In: Rai, L.C., Gaur, J.P. (Eds.) Algal Adaptation to Environmental Stresses, pp. 427. Springer-Verlag, Berlin, Heidelberg.

Guillard, R. 1973. Methods for microflagellates and nannoplankton. In: Stein, J.R. (Ed.) Culture Methods and Growth Measurements: 69-85. Cambridge University Press, Cambridge.

Hallegraeff, G.M. 2010. Algae of Australia. Phytoplankton of temperate waters. In: Hallegraeff, G.M., Bolch, C.J.S., Hill, D.R.A., Jameson, I, LeRoi, J.-M., McMinn, A., Murray, S., de Salas, M.F., Saunders, K. (Eds.) pp. 1-15. Canberra \& Melbourne: ABRS; CSIRO Publishing.

Hanley, D.E., Bourassa, M.A., O'brien, J.J., Smith, S.R., Spade, E.R. 2003. A quantitative evaluation of ENSO índices. Journal of Climate 16: 1249-1258.

Hernández-Becerril, D.U. 2000. Morfología y taxonomía de algunas especies de diatomeas del género Coscinodiscus de las costas del Pacifico Mexicano. Revista de Biología Tropical 48(1): 7-18.

Hernández-Becerril, D.U. 2014. Biodiversidad de algas planctónicas marinas (Cyanobacteria, Prasinophyceae, Euglenophyta, Chrysophyceae, Dictyochophyceae, Eustigmatophyceae, Parmophyceae, Raphidophyceae, Bacillariophyta, Cryptophyta, Haptophyta, Dinoflagellata) en México. Revista Mexicana de Biodiversidad (85): 4453.

Hernández-Pérez, A., Labbé, J.I. 2014. Microalgas, cultivo y beneficios. Revista de Biología Marina y Oceanografía 49(2): 157-173.

Infante, C., Angulo, E., Zarate, A., Flores, J., Barrios, F., Zapata, C. 2012. Propagación de la microalga Chlorella sp. en cultivo por lote: cinética de crecimiento celular. Avances en Ciencias e Ingeniería 3(2): 159-164. 
Islam, M.A., Magnusson, M., Brown, R.J., Ayoko, G.A., Nabi M.N., Heimann, K. 2013. Microalgal species selection for biodiesel production based on fuel properties derived from fatty acid profiles. Energies 6: 5676-5702.

Kaden, J., Brùckner, S.I., Machill, S., Krafft, C., Pòppl, A., Brunner, F. 2017. Iron incorporation in biosilica of the marine diatom Stephanopyxis turris: dispersed or clustered? Biometals 30: 71-82.

Leal, S., Medina, M.A., Guerrero, A., Piña, P., Nieves, M., Curbelo, R. 2013. Concentración y composiciones orgánica y proximal de dos especies de diatomeas bentónicas a diferentes salinidades. Universidad y Ciencia Trópico Húmedo 29(1): 45-52.

Lemus, N., Urbano, T., Arredondo-Vega, B.O., Guevara, M., Vásquez, A., Carreón-Palau, L., Vallejo, N. 2006. Growth and biochemical profile of Chaetoceros muelleri cultured in batch and semicontinuous systems. Ciencias Marinas 32(3): 597-603.

López-Elías, J.A. 2002. Evaluación cualitativa y cuantitativa de los sistemas de producción de microalgas en seis laboratorios comerciales del noroeste de México. Tesis de Doctorado, Facultad de Ciencias marinas, Universidad de Colima. Colima. URL: http://digeset.ucol.mx/tesis_posgrado/Pdf/ Jose\%20Antonio\%20Lopez\%20Elias.pdf

Matsumoto, M., Mojima, D., Nonoyama, T., Ikeda, K., Maeda, Y., Yoshino, T., Tanaka, T. 2017. Outdoor cultivation of marine diatoms for year-round production of biofuels. Marine Drugs 15(4): 94.

Mcginnis, K.M., Dempster, T.A., Sommerfeld, M.R. 1997. Characterization of the growth and lipid content of the diatom Chaetoceros muelleri. Journal of Applied Phycology 9: 19-24.

Meave-del Castillo, M.E., Zamudio-Resendiz, M.E., CastilloRivera, M. 2012. Riqueza fitoplanctónica de la Bahía de Acapulco y zona costera aledaña, Guerrero, México. Acta Botánica Mexicana 100: 405-487.

Moreno, J., Licea, S., Santoyo, H. 1996. Diatomeas del Golfo de California. Universidad Autónoma de Baja California Sur, Baja California. 273 pp.

Moreno-Díaz, G., Rojas-Herrera, A.A., González-González, J., Violante-González, J., Rosas-Acevedo, J., García-Ibáñez, S. 2015. Temporal variation in the abundance and composition of phytoplankton species, collected with net in the Acapulco Bay, Mexico. Biociencias 3(2): 88-102.

Moronta, R., Mora, R., Morales, E. 2006. Respuesta de la microalga Chlorella sorokiana al $\mathrm{pH}$, salinidad y temperatura en condiciones axénicas y no axénicas. Revista de la Facultad de Agronomía 23: 27-41.

Nájera-Arce, C., Álvarez-Fitz, P., Pérez-Castro, D., Toribio-Jiménez, J., Castro-Alarcón, N. 2018. Actividad antibacteriana de diatomeas marinas aisladas de Acapulco, Guerrero, México. Revista de Biología Marina y Oceanografía 53(2): 195-207.

Ojeda-Rodríguez, A. 2003. Diatomeas centrales del orden Triceratiales en aguas costeras de las islas Canarias. Vieraea 31: 207-217.

Panta-Vélez, R.P., Macay-García, A.G., Moncayo-Zambrano, E.M., Vélez-Chica, J.C. 2016. Growth of Chaetoceros gracilis and Isochrysis galbana microalgae with agricultural fertilizers, in the laboratory. La Técnica 16: 44-55.

Pérez, C.D. 1995. Cultivo Experimental de las Diatomeas Thalassiosira subtilis, Skeletonema costatum y Chaetoceros affinis en condiciones de laboratorio para fines de acuacultura. Tesis de Maestría, Facultad de Ciencias marinas, Universidad de Colima. Colima. URL: http:// digeset.ucol.mx/tesis_posgrado/Pdf/PDonaciano.pdf

Porta-Vales, A. 2009. Optimización del cultivo de diatomeas bentónicas para el cultivo de post-larvas de abalón (Haliotis tuberculata coccinea Reeve, 1846). Tesis de maestría, Instituto Canario de Ciencias Marinas, España. 102 pp. URL: https://acceda.ulpgc.es/ bitstream/10553/8707/5/0668996_00000_0000.pdf

Renaud, S.M., Luong-Van, T., Lambrinidis, G., Parry, D.L. 2002. Effect of temperature on growth, chemical composition and fatty acid composition of tropical Australian microalgae grown in batch cultures. Aquaculture 211: 195-214.

Richmond, A. 2004. Handbook of microalgal culture: biotechnology and applied phycology. Blackwell Science, Oxford. 566 pp.

Rojas-Herrera, A.A., Violante-González, J., García-lbáñez, S., Sevilla-Torres, V.M.G, Gil-Guerrero, J.S., Flores-Rodríguez, P. 2012. Temporal variation in the phytoplankton community of Acapulco Bay, Mexico. Microbiological Research 3(4): 13-19.

Round, F.E., Crawford, R.M., Mann, D.G. 2007. Diatoms: Biology and morphology of the genera. Cambridge University Press, Cambridge. 350-351 pp.

Sarthou, G., Timmermans, Kr, Blain, S., Tréguer, P. 2005. Growth physiology and fate of diatoms in the ocean: a review. Journal of Sea Research 53(1-2): 25-42.

Thompson, P.A., Guo, M., Harrison, P.J., Whyte, J.N.C. 1992. Effects of variation in temperature: On the fatty acid composition of eight species of marine phytoplankton. Journal of Phycology 28: 488-497.

Vásquez, S.A, Guevara, M., González, M., Cortez, R., ArredondoVega, B.O. 2013. Crecimiento y composición bioquímica de Thalassiosira pseudonana (Thalassiosirales: Thalassiosiraceae) bajo cultivo semi-continuo en diferentes medios y niveles de irradiancias. Biología 
Tropical 61(3): 1003-1013.

Vásquez, S.A., Miguel, G., González, M., Lemus, N., ArredondoVega, B.O. 2010. Growth and biochemical composition of Skeletonema costatum (Greville 1866) Cleve, 1878 (Heterokontophyta: Bacillariophyceae) in function of irradiance and culture medium. Saber 22(2): 149-159.

Zafra-Trelles, A.M., Díaz, B.M.E., Dávila, G.F.A., Bopp, V.G.M., Vela, K.A., López, E.M.B., Castillo, G.J.B., Colchado,
C.J.P.E. 2017. Cultivo de microalgas marinas potenciales para la acuicultura del litoral entre Puerto Salaverry y Puerto Chicama. Arnaldoa 24(2): 567-582.

Zaouri, N., Gutierrez, L., Dramas, L., Garces, D., Croue, J.P. 2017. Interfacial interactions between Skeletonema costatum extracellular organic matter and metal oxides: Implications for ceramic membrane filtration. Water Research 116: 194-202.

Received: 13.05.2019

Accepted: 04.02.2020 